\title{
The Norton Dome and the Nineteenth Century Foundations of Determinism
}

\author{
By: Marij van Strien
}

In this paper, I connect the contemporary discussion in philosophy of physics about the Norton dome with nineteenth century literature on similar physical systems, and I show that what is currently the standard conception of determinism in classical physics was not generally accepted in nineteenth century France.

We usually consider classical mechanics as the prime example of a deterministic theory, but Norton has shown in 2003 that there are possible systems in classical mechanics which are not deterministic, notably the system which has become known as the Norton dome. This is regarded as a newly discovered instance of indeterminism in classical physics and it has raised quite some discussion among philosophers of physics in the last few years. ${ }^{1}$ However, I show that the same instance already appeared during the nineteenth century. I discuss four nineteenth-century French authors who wrote about such systems, namely Siméon Denis Poisson, Jean-Marie Duhamel, Joseph Boussinesq and Joseph Bertrand, and examine their ideas about determinism.

The kind of indeterminism that is at issue in Norton's dome can be called "Lipschitzindeterminism", and amounts to the fact that the differential equations which are the equations of motion of a certain system may not have a unique solution for given initial conditions. The Norton dome is a system consisting of a mass point on top of a dome of a particular shape, for which it can be shown that the mass point can roll off at an undetermined time. In an article about the Norton dome, Malament discusses also another possible Lipschitz-indeterministic system in which a particular force acting on a point particle leaves the motion of the particle undetermined. ${ }^{2}$

These systems violate what is currently our standard conception of determinism in classical physics, namely the statement that for each system in classical physics, there are differential equations of motion of the form $d^{\wedge} 2 r / d t^{\wedge} 2=F(r)$, which, together with the initial conditions $r(t 0)=r 0$ and dr/dt $(t 0)=$ v0 uniquely determine the future states of the system. This is the formulation of determinism in classical physics that is used in most contemporary literature. ${ }^{3}$ In this way, determinism can be understood as a theorem in classical physics, rather than as a metaphysical conviction, such as the idea that every event has a cause.

In this paper I consider whether the fact that Lipschitz-indeterministic systems were known in the nineteenth century (though not under this name) means that determinism in physics was not universally accepted at that time. I argue that the example itself did not convince many people that there was indeterminism in physics. The reason for this is that the authors which I discuss employed alternative conceptions of determinism in physics which were not necessarily violated by Lipschitz-indeterministic systems.

In 1806, Poisson discussed the possibility that the equations of motion for a physical system do not have a unique solution. ${ }^{4}$ Of the two examples that he discussed, one was analogous to the recent one by Malament, in which a particular force acting on a point particle leaves the motion of the particle undetermined. Poisson emphasized that in these cases, one had to find out which solution was the 'right' one; this involved additional physical considerations which were not captured in the laws of motion. The issue was treated in a largely similar way by Duhamel in $1845 .{ }^{5}$ For both, determinism was 
not equal to the statement that there was for each system a set of differential equations with a unique solution, for it might be that the equations had several solutions without there being a failure of determinism. Therefore, the possibility of Lipschitz-indeterministic systems did not imply that there was actual indeterminism.

Lipschitz-indeterministic systems played an important role in the work of Boussinesq, who, in 1878, discussed several such systems, one of which was similar to the Norton dome. ${ }^{6}$ Boussinesq used these cases of physical indeterminism as the foundation of an elaborate theory about free will and organization in living organisms, a theory which drew quite some attention at the time.

Boussinesq attributed great significance to the fact that the equations of motion could have non-unique solutions, yet he regarded these equations themselves as approximations or idealizations of what happens in reality. In a criticism of Boussinesq's theory, the famous mathematician Joseph Bertrand showed that this made Boussinesq's theory highly problematic. ${ }^{7}$ Bertrand himself also argued that differential equations such as the equations of motion are approximations of what really happens. He argued that therefore, the indeterminism that these equations can exhibit does not have to be reflected in real physical systems. Bertrand's conviction that there was determinism was not grounded in the theorem that there are for each system differential equations which have a unique solution, but was rather a metaphysical conviction.

In the contemporary literature on Norton's dome, there has been a debate about the idealizations on which the example depends, such as the assumption that the dome is infinitely smooth and rigid and that the particle can move frictionless over the surface of the dome. Korolev for example has argued that the resulting indeterminism is no more than an artefact of such idealizations. ${ }^{8}$ This has triggered some discussion about which idealizations are "allowed" in Newtonian mechanics. This discussion centres on the properties of the theory rather than on what is possible in reality. The discussion between Boussinessq and Bertrand, however, shows that for them there were more fundamental issues involved about the relation between mathematics and reality, as they argued that differential equations in general involved idealizations.

To conclude, Lipschitz-indeterministic systems only imply a violation of determinism if determinism is defined as the statement that in a classical system, the differential equations of motion are rigorously valid and these equations alone determine the future states of the system. Though this statement is now our standard conception of determinism in classical physics, it was still in a state of development during the nineteenth century. The authors that I discuss diverged from this definition in various ways and had various alternative conceptions of determinism. Though determinism as a general metaphysical principle was seldom doubted, it was not necessarily founded on a physical theorem about the uniqueness of solutions to certain differential equations.

\section{Footnotes:}

1 See, among others: - Norton, John (2003): Causation as Folk Science. Philosopher'sImprint 3 (4); Norton, John (2008): The dome: an unexpectedly simple failure of determinism. Philosophy of Science 75 (2008): 786-98; Phil. of Sci. Assoc. 20th Biennial Mtg. (Vancouver): PSA 2006 Symposia; - Malament, David B. (2008): Norton's Slippery Slope. Philosophy of Science 75 (2008), pp. 799-816; - Wilson, Mark (2009): Determinism and the mystery of the missing physics. British Journal for the Philosophy of Science 60 (2009), pp. 173-193; - Korolev, Alexandre (2010): The Norton-type Lipschitz-indeterministic systems and elastic phenomena: Indeterminism as an artefact of infinite idealizations. In: [2008] Philosophy of Science Assoc. 21st Biennial Mtg (Pittsburgh, PA) : PSA 2008 Contributed Papers; - 
Zinkernagel, Henrik (2010): Causal fundamentalism in physics. In: Suárez, Dorato \& Redei (eds.), EPSA philosophical issues in the sciences: Launch of the European philosophy of science association.

Dordrecht: Springer, 2010.

2 See Malament (2008).

3 See Earman, John (1986): A primer on determinism. Dordrecht: Reidel, p. 30; Malament (2008)

4 Poisson (1806): Mémoire sur les solutions particulières des équations différentielles et des équations aux différences. Journal de l'École Polytechnique, XIII e cahier, t. VI., 1806, pp. 60-125.

5 Duhamel (1845):Cours de mécanique de l'école polytechnique, tome I. Paris: Bachelier, 1845.

6 Boussinesq (1879): Conciliation du véritable déterminisme mécanique avec l'existence de la vie et de la liberté morale. Mémoires de la société des sciences, de l'agriculture et des arts de Lille, 1879, t. VI, 4e série, pp. 1-257.

7 Bertrand (1878) - Conciliation du véritable déterminisme mécanique avec l'existence de la vie et de la liberté morale, par J. Boussinesq. Journal des Savants, 1878, pp. 517-523.

8 Korolev (2010). 